\title{
Management of Xerostomia Patient with Salivary Reservoir Designed in Upper Complete Denture and Lower Cast Partial Denture
}

\author{
${ }^{1}$ Vimal Arora, ${ }^{2}$ Dinesh Kumar, ${ }^{3}$ Virender Singh Legha, ${ }^{4}$ KV Arun Kumar
}

\begin{abstract}
Xerostomia is a common complaint found often among older adults. Dry mouth has multiple oral health consequences and affects quality of life. It can produce serious negative effects on the patient's quality of life by affecting dietary habits, nutritional status, speech, taste and tolerance to dental prosthesis and increasing the risk of oral infection, including candidiasis and susceptibility to dental caries, periodontal disease and tooth loss. Saliva protects oral mucosa and teeth against harmful substances, lubricates the mouth to facilitate chewing, swallowing and speech, and reduces tissue trauma. Presence of adequate amounts of saliva within denture and tissue interface is essential. Without enough saliva, a denture will inadequately adhere to tissues, partly through loss of surface tension. Sometimes, the cause may easily be eliminated but, in many instances, it is not possible, and the condition is persistent and often progressive. There are several approaches to manage dry mouth. In this article, a palatal reservoir design for upper complete denture and lower cast RPD with salivary reservoir has been described, which completely satisfies the patient need for long time.
\end{abstract}

Keywords: Xerostomia, Radiotherapy, Salivary reservoir, Complete denture, Artificial saliva.

How to cite this article: Arora V, KumarD, Legha VS, Kumar KVA. Management of Xerostomia Patient with Salivary Reservoir Designed in Upper Complete Denture and Lower Cast Partial Denture. J Contemp Dent 2014;4(1):56-59.

Source of support: Nil

Conflict of interest: None

\section{INTRODUCTION}

Xerostomia is defined as dry mouth resulting from decreased salivary flow and is manifested as increased caries incidence, gingivitis, oral candidiasis and burning mouth. Saliva acts as a primary defensive factor in oral cavity ${ }^{1}$ and a reduction in

${ }^{1}$ DGDS and Colonel Commandant, ${ }^{2}$ Assistant Professor,

${ }^{3,4}$ Reader and Instructor

${ }^{1}$ Director General Dental Services, Army Dental Corps, Adjutant General's Branch, New Delhi, India

${ }^{2}$ Department of Dental Surgery and Prosthodontics, Armed Forces Medical College, Pune, Maharashtra, India

${ }^{3,4}$ Department of Dental Surgery, Armed Forces Medical College, Pune, Maharashtra, India

Corresponding Author: Dinesh Kumar, Assistant Professor Department of Dental Surgery and Prosthodontics, Armed Forces Medical College, Pune, Maharashtra, India, Phone: 02026306299, e-mail: drdineshsaini@yahoo.co.in its flow rate is associated with oral and pharyngeal disorders and requires early diagnosis and intervention.

Xerostomia is not a disease, but it may be a symptom of various medical conditions, a side effect of a radiation to the head and neck, or a side effect of a wide variety of medications. Drugs are the most common cause of xerostomia in elderly people because these medications adversely affect salivary function. ${ }^{2}$ Patients with xerostomia often complain of dry mouth, difficulty in eating, speaking and swallowing. ${ }^{3,4}$

Depending upon the cause, various treatment options are available; like in medication induced xerostomia, change in dosage/timing or prescribing drug substitute may reduce the severity of the problem. Gustatory or salivary stimulation of the salivary glands by use of sugar free chewing gums or lozenges or citrus juices is also helpful in cases of lowered salivary gland function. If damage is permanent, symptomatic treatment is required which includes the change in dietary pattern, frequent sipping of water and use of artificial salivary substitutes. ${ }^{5,6}$

In case of partially or completely edentulous patients, presence of adequate amounts of saliva between the denture/tissue interfaces is essential. The physical factors like adhesion, cohesion and interfacial surface tension which play an important role in retention of denture will not be effective in absence of a thin film of saliva between denture and the soft tissues they rest upon. In addition, tissues contacting a denture will become chafed and irritated without the lubricating effects of saliva. To overcome all these symptoms, salivary reservoirs must be included in the prosthesis. Various reservoir designs have been discussed in literature to incorporate reservoirs in the dentures either by modifying the palatal region of maxillary dentures ${ }^{7,8}$ or by creating chambers in the bulky areas of dentures. ${ }^{9,10}$ This paper presents rehabilitation of a case of xerostomia with salivary reservoirs designed in upper complete denture and lower cast partial denture.

\section{CASE REPORT}

A 70 years old patient was referred from the department of surgery for prosthetic rehabilitation. Patient's chief complaint was inability to chew, difficulty in swallowing and dryness of mouth for last 06 months. From his medical 
documents it was found that patient was diagnosed as a case of malignant lesion and had undergone alveolectomy in right side in premolar region keeping the lower border of mandible intact. This was followed by 04 radiotherapy sessions which finished 06 months back. On clinical examination, it was found that his all maxillary teeth and 43 to 45 were missing and his oral hygiene was poor with severe halitosis. Oral prophylaxis was performed along with education and motivation for maintenance of oral hygiene. Dentoalveolar defect was lined with mucosa and completely healed. The patient was taken up for rehabilitation by making upper complete denture with salivary reservoir and lower cast partial denture with reservoir to overcome the problem of dryness of mouth. In upper complete denture the salivary reservoir was incorporated in the palatal region and in lower cast partial denture the salivary reservoir was incorporated in edentulous region by making the edentulous portion hollow.

Maxillary denture was fabricated in conventional manner. After fabrication of complete denture, the palatal potion was thinned out and zinc-oxide eugenol paste was applied which act as a spacer (Fig. 1). Over the spacer of ZOE paste, a sheet of modeling wax was adapted and this sheet was processed in heat polymerized resin (Fig. 2). Now the sheet was attached to the upper complete denture after removing the spacer with the help of autopolymerizing resin. Two small openings were created in this acrylic plate (Fig. 3). The artificial saliva was filled with the help of syringe (Fig. 3).

For fabrication of mandibular cast partial denture, all the conventional steps were followed till the fabrication of refractory cast. Because extra space was present in lower right region, the space was utilized to fabricate a hollow reservoir. The wax pattern was made in conventional manner with linguplate as major connector, following all the principals of cast partial design in edentulous region a chamber was created in wax. The wax pattern was invested and $\mathrm{Co}-\mathrm{Cr}$ framework was fabricated in conventional manner (Fig. 4). To convert the open chamber into a closed reservoir, a closely fitting lid of the chamber was fabricated by adapting a wax sheet over the margins of the open chamber. This sheet was again invested, casted and finished in conventional manner. The close fitting of the lid was checked onto the open chamber of the framework. Over the chamber lid, occlusal rim was fabricated, jaw relation recorded and teeth arrangement was done. The detachable part of framework covering the salivary reservoir was processed separately along with artificial teeth. Detachable portion now was fitted on to the cast partial denture framework (Fig. 5). Two holes were created, one on the lingual surface and other on the buccal surface of chamber for flow of saliva. The patient was trained to fill up the saliva in the salivary reservoirs fabricated in upper complete denture and hollow chamber created in lower cast partial denture.

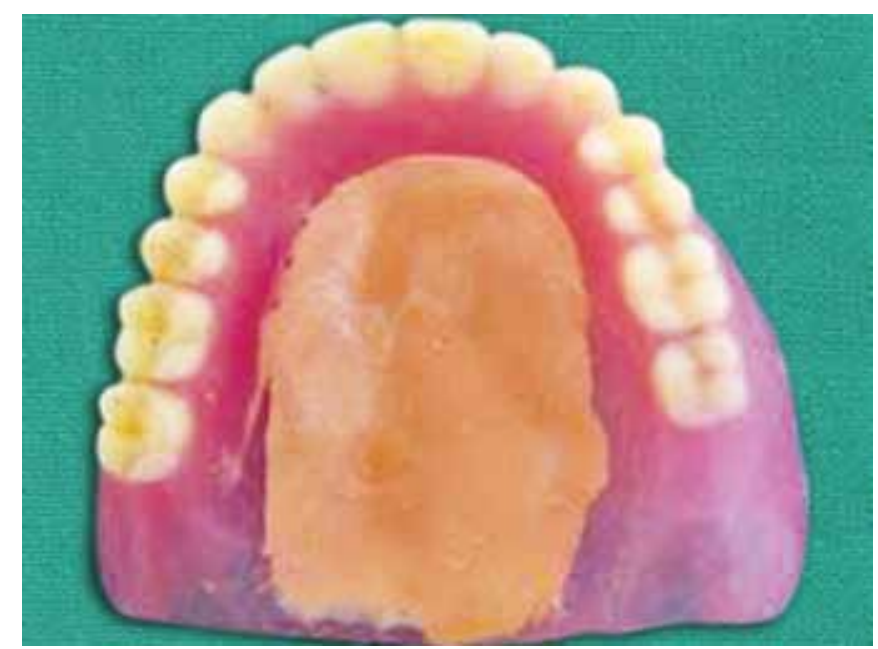

Fig. 1: Application of spacer on the palatal surface of denture

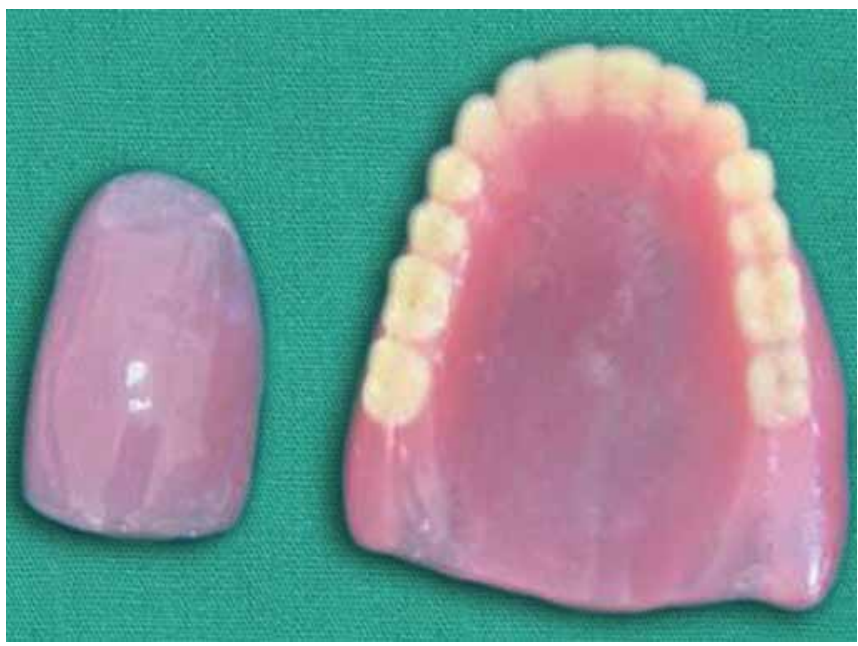

Fig. 2: The reservoir lid processed in heat cure acrylic resin

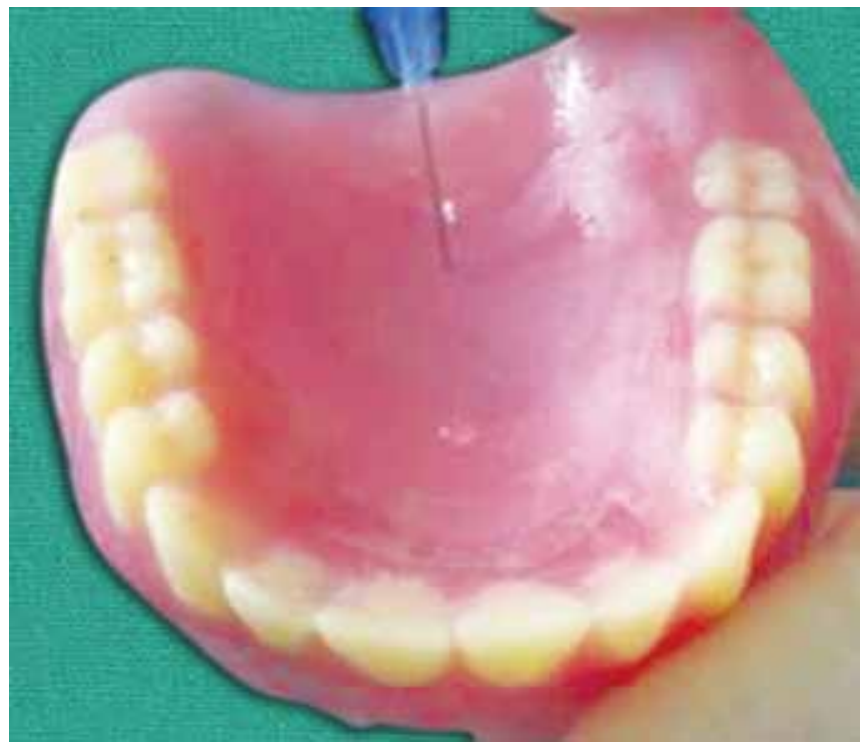

Fig. 3: Salivary substitute injected into the reservoir 


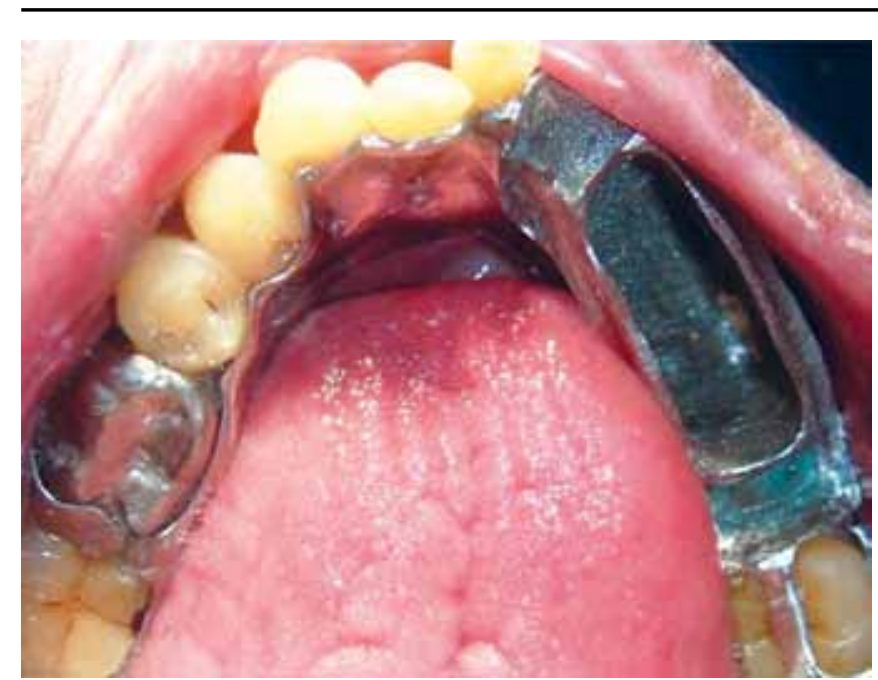

Fig. 4: Finished framework in the oral cavity

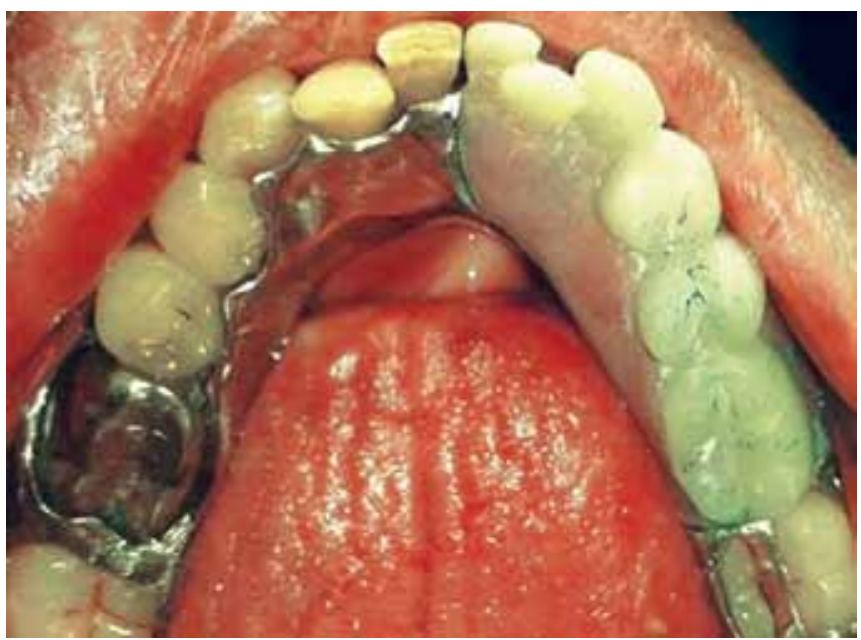

Fig. 5: Cast partial denture with detachable artificial teeth

\section{DISCUSSION}

The average daily production of whole saliva in healthy individual varies between 1 and $1.5 \mathrm{~L}$. The normal flow rate for unstimulated saliva is 0.3 to $0.5 \mathrm{ml} / \mathrm{min}$ and for the stimulated saliva is 1 to $2 \mathrm{ml} / \mathrm{min}$. Unstimulated flow of saliva is mainly produced from submandibular gland contributing to $65 \%$ of total amount, $20 \%$ is secreted by parotid, 7 to $8 \%$ from sublingual and approximately $10 \%$ from minor salivary glands whereas stimulated flow of saliva is mainly from parotid contributing more than $50 \%$ of total salivary secretions. ${ }^{4}$ Values less than $0.1 \mathrm{ml} / \mathrm{min}$ are typically considered as xerostomia.

There are two types of xerostomia described in literature. Primary xerostomia - in this case, a pathologic lesion is always present in the salivary glands as a manifestation of either localized or systemic disease. Secondary xerostomiawhen no salivary lesion is present and reduced production of saliva is because of some other factors like medications, radiation exposure, systemic illnesses, chronic mouth breathing and inadequate fluid consumption, dehydration through hemorrhage, sweating, diarrhea, vomiting, polyuria or diabetes mellitus or insipidus, various vitamin and hormonal deficiencies and anemia.

Radiation therapy: Unfortunately the salivary gland tissue that is included in the field of radiation suffers a permanent loss of function, usually within the first 2 weeks of fractionated radiation treatment. The reasons for the high radiosensitivity of salivary tissues are not well understood, but it may be due to damage to the blood supply (endarteritis), interference with nerve transmission, or destruction of the gland itself. The effects of radiation are dose, time and field dependent. The parotid glands are the most radiosensitive, followed by the submandibular, sublingual and minor glands. ${ }^{11}$

Management of these patients begins with thorough patient education. Patients should undergo nutritional counseling to limit the harmful effects of reactionary diet modifications and should be encouraged to chew because mechanical stimulation of periodontal receptors and stimulation of the tongue and oral mucosa are vital stimuli for salivation.

Different types of artificial saliva are available that alleviate the feeling of dryness for longer period of time than mouth rinses. If saliva secretion cannot be stimulated, symptomatic treatment involves the use of saliva substitutes. Commercially available saliva substitutes containing thickening agents, such as carboxymethyl cellulose or mucin, are the most common. Recently, saliva substitutes based on biotene and mucopolysaccharides have been developed and are recommended for patients with extremely low salivary production rates.

Intraoral saliva reservoir in the hollowed lingual flange of mandibular denture can be fabricated. Even in the maxillary denture, it can be fabricated in the palatal area. This procedure can give a period of continuous wetting for at least 2 hours. The period varies with the degree of xerostomia and volume of reservoir. The palatal surface of the denture is thicken with the plastic wax, other mouth temperature waxes like soft beeswax or plastic wax can also be used for contouring the palatal form. Unacceptable distortions of speech must be prevented. Two release holes are made, one in the rugae area and another in the posterior part of the palate. The release holes of size of $0.2 \mathrm{~mm}$ are made with a small diamond bur. Salivary substitute is injected with the help of a syringe and the patient is trained to load the reservoir. Instructions are given for proper hygiene of the oral cavity and the denture. The internal surface of the reservoir is cleaned by forcefully injecting the water through the openings every day with water and once in 15 days with $7 \%$ sodium hypochlorite solution to prevent any growth of microorganisms. 


\section{CONCLUSION}

Xerostomia is a common problem and if not recognized and treated, can have a significant effect on a patient's quality of life. Retention of denture is a highly complex one and the physical properties like surface tension, adhesion and cohesion are only effective if a thin film of saliva is present. Without saliva, denture wearing becomes a painful experience. Addition of salivary reservoirs in prosthesis can improve the quality of life of the complete and partially edentulous patients.

\section{REFERENCES}

1. Mandel ID. Role of saliva in maintaining oral homeostasis. J Am Dent Assoc 1989;119:298-304.

2. Greenspan D. Xerostomia: diagnosis and management. Oncology 1996;10(Suppl):7-11.

3. Edgar WM. Saliva: its secretion, composition and functions. $\mathrm{Br}$ Dent J 1992;172:305-312.
4. Williamson RT. A review of saliva: normal composition flow, and function. J Prosthet Dent 2001;85:162-169.

5. Vergo TJ Jr, Kadish SP. Dentures as artificial saliva reservoirs in the irradiated edentulous cancer patient with xerostomia: a pilot study. Oral Surg Oral Med Oral Pathol 1981;51:229-233.

6. Sinclair GF, Frost PM, Walter JD. New design for an artificial saliva reservoir for the mandibular complete denture. J Prosthet Dent 1996;75:276-280.

7. Huisman MC, Vissink A, Gravenmade EJ. Construction of an artificial saliva reservoir in an existing maxillary denture. J Prosthet Dent 1986;56:70-74.

8. Toljanic JA, Schweiger JW. Fabrication of an artificial saliva reservoir denture system for xerostomia management. Quintessence Dent Technol 1985;9:355-358.

9. Posti J, Makila E, Hirvikangas M. Treatment of xerostomia through use of dentures containing reservoirs of saliva substitute. Proc Finnish Dent Soc 1989;85:47-50.

10. Vissink A, Gravenmade EJ, Panders AK, Olthof A, Vermey A, Huisman MC, Visch LL. Artificial saliva reservoirs. J Prosthet Dent 1984;52:710-715.

11. Sreebny LM. Saliva in health and disease: an appraisal and update. Int Dent J 2000;50:140-161. 